\title{
Breaking a Robust Remote User Authentication Scheme Using Smart Cards
}

\author{
Ding Wang ${ }^{1,2}$, Chun-guang $\mathrm{Ma}^{1}$, Sen-dong Zhao ${ }^{1}$, and Chang-li Zhou ${ }^{1}$ \\ ${ }^{1}$ College of Computer Science and Technology, Harbin Engineering University \\ 145 Nantong Street, Harbin City 150001, China \\ wangdingg@mail.nankai.edu.cn \\ ${ }^{2}$ Automobile Management Institute of PLA, Bengbu City 233011, China
}

\begin{abstract}
Understanding security failures of cryptographic protocols is the key to both patching existing protocols and designing future schemes. Recently, Yeh et al. showed that Hsiang and Shih's password-based remote user authentication scheme is vulnerable to various attacks if the smart card is nontamper resistant, and proposed an improved version which was claimed to be efficient and secure. In this study, however, we find that, although Yeh et al.'s scheme possesses many attractive features, it still cannot achieve the claimed security goals, and we report its following flaws: (1) It cannot withstand offline password guessing attack and key-compromise impersonation attack under their non-tamper resistance assumption of the smart card; (2) It fails to provide user anonymity and forward secrecy; (3) It has some other minor defects. The proposed cryptanalysis discourages any use of the scheme under investigation in practice. Remarkably, rationales for the security analysis of password-based authentication schemes using smart cards are discussed in detail.
\end{abstract}

Keywords: Cryptanalysis, Authentication protocol, Offline password guessing attack, Smart card, Forward secrecy.

\section{Introduction}

With the development of distributed computer networks, it is easy for user terminals to share information and computing power with hosts [1,2]. The distributed locations of service providers make it efficient and convenient for subscribers to access the resources, and it is of great concern to protect the systems and the users' privacy and security from malicious adversaries. Accordingly, user authentication becomes an essential security mechanism for remote systems to assure one communicating party of the legitimacy of the corresponding party by acquisition of corroborative evidence. Among numerous methods for user authentication, password based authentication with smart cards is one of the most promising techniques and has been widely adopted over insecure networks to validate the legitimacy of users.

In 1981, Lamport [3] introduced the first password authentication scheme to authenticate a remote user over an insecure channel. This seminal scheme was later refined and used in a number of applications, notably Haller's famous S/KEY onetime password system [4]. Later on, Chang and $\mathrm{Wu}$ [5] introduced the smart cards 
into remote user authentication schemes, since then there have been many smart card based password authentication schemes proposed [6-10]. In such schemes, the user is equipped with a smart card and a password as identification verifiers. When the user wants to login to the server, she provides the card with her password, which is used to construct a login request that is sent to the server. Upon receiving the request, the server authenticates these messages and provides the desired service if the verifiers are found valid. If mutual authentication occurs, the client is also convinced that the corresponding server is authentic. More admired schemes also achieve session key agreement for securing the subsequent data communications.

The common adversary model to evaluate the security of authentication protocols using smart cards assumes an attacker with full control over the communication channel between the user and the remote server [7,10,11]. Accordingly, all the messages exchanged can be blocked, intercepted, deleted, or modified by the attacker, and the attacker can also insert his/her own fabricated messages. Secondly, protocols must assume that the attacker can temporarily get access to the legitimate user's smart card, which is reasonable in practice. What's more, since recent research results have shown that the secret data stored in the common smart card could be extracted by some means, such as monitoring the power consumption [12,13] or analyzing the leaked information [14], the smart card should be assumed to be non-tamper resistant, i.e., the secret information stored in the smart card can be revealed.

As mentioned in $[10,15]$, a sound password authentication scheme should be able to withstand a number of sophisticated distinct types of attacks, such as replay attack, password guessing attack, parallel session attack, denial of service attack, stolen verifier attack, and user/server impersonation attack. As resistance to these passive and active attacks is a basic security requirement for authentication protocols, the following desirable attributes are also of great importance in the case of an authentication scheme with session key establishment [16,17]:

$i$. Resistance to known key attack. A protocol still achieves its security goal in the face of an adversary who has learned some previous session keys.

ii. Provision of forward secrecy. Even if long-term private key of one or more entities are compromised, the secrecy of previous session keys is not affected.

iii. Resistance to unknown key-share attack. The entity $i$ cannot be coerced into sharing a key with entity $j$ without $i$ 's knowledge, i.e., when $i$ believes the key is shared with some other entity $k$, where $k \neq j$.

$i v$. Resistance to key-compromise impersonation attack. It is desirable that the leakage of entity $i$ 's long term private key does not enable an adversary to impersonate other entities to $i$.

In 2009, Hsiang and Shih [8] showed that Yoon et al.'s scheme [6] is susceptible to user impersonation attack, offline password guessing attack and parallel session attack. To overcome these defects, Hsiang and Shih presented an enhanced version. Later on, Sood et al. [9] showed that Hsiang and Shih's scheme still suffers from offline password guessing attack and user impersonation attack, and user anonymity is not preserved. More recently, Yeh et al. [18] identified that, besides the security flaws found by Sood et al., Hsiang and Shih's scheme is also prone to undetectable online password guessing attack. Consequently, Yeh et al. proposed a further improved 
version to eliminate the aforementioned security flaws. In this paper, however, we will demonstrate that Yeh et al.'s scheme is still vulnerable to the offline password guessing attack and key-compromise impersonation attack. Moreover, their scheme fails to provide the property of forward secrecy and user anonymity.

The remainder of this paper is organized as follows: in Section 2, we briefly review Yeh et al.'s authentication scheme. Section 3 describes the weaknesses of Yeh et al.'s scheme. Finally, we conclude this paper in the last section.

\section{$2 \quad$ Review of Yeh et al.'s Scheme}

In this section, we briefly review the first scheme, i.e. the improvement on Hsiang and Shih's scheme, proposed by Yeh et al. in [18]. Their scheme, summarized in Fig.1, consists of four phases, namely, the registration phase, the login phase, the verification phase and password update phase. For ease of presentation, we employ some intuitive abbreviations and notations listed in Table 1.

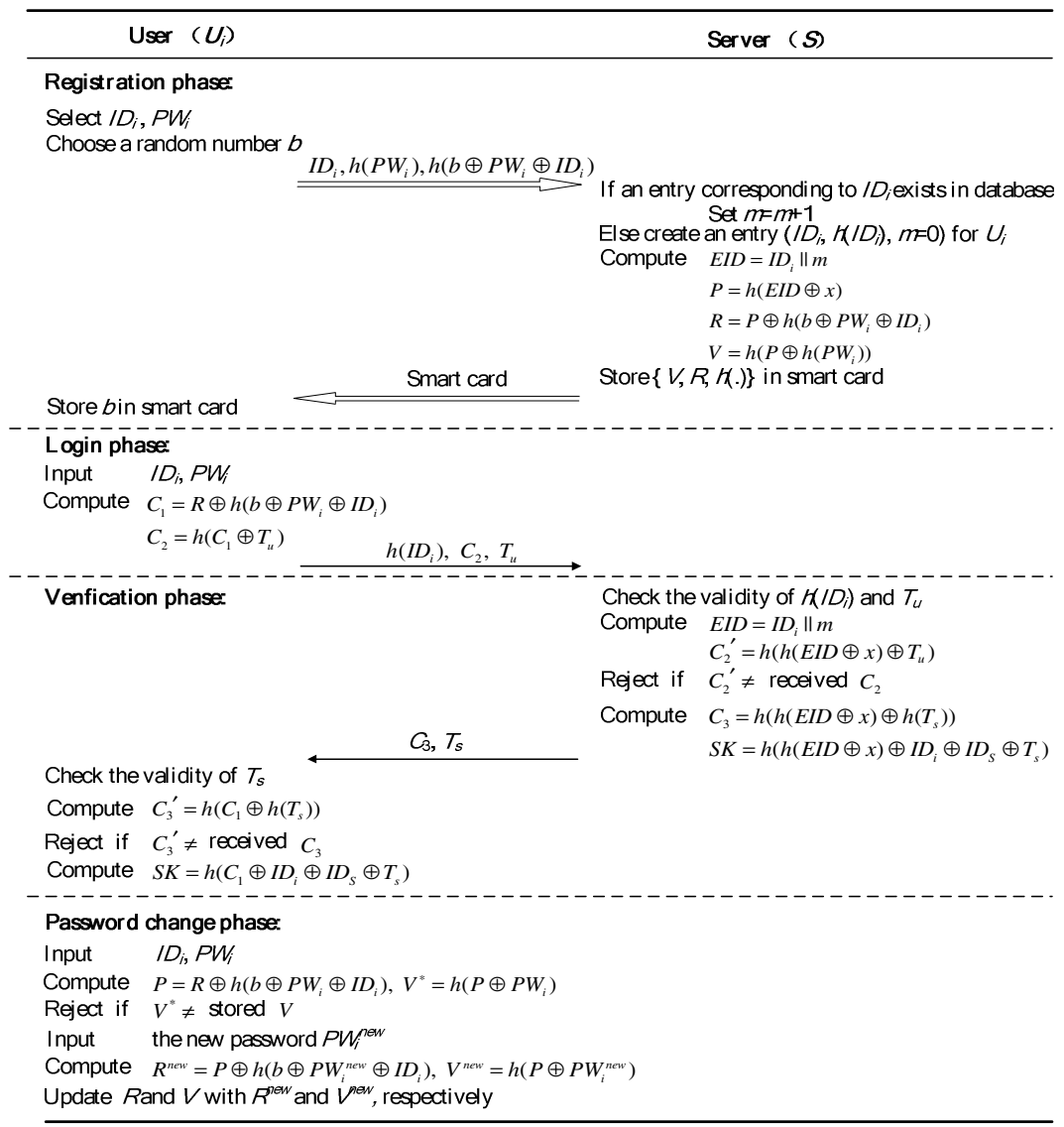

Fig. 1. Yeh et al.'s remote user authentication scheme 
Table 1. Notations

\begin{tabular}{ll}
\hline Symbol & Description \\
\hline$U_{i}$ & $i^{\text {th }}$ user \\
$S$ & remote server \\
$I D_{i}$ & identity of user $U_{i}$ \\
$P W_{i}$ & password of user $U_{i}$ \\
$x$ & the secret key of remote server $S$ \\
$h(\cdot)$ & collision free one-way hash function \\
$\oplus$ & the bitwise XOR operation \\
$\|$ & the string concatenation operation \\
$A \Rightarrow B: M$ & message $M$ is transferred through a secure channel from $A$ to $B$ \\
$A \rightarrow B: M$ & message $M$ is transferred through a common channel from $A$ to $B$ \\
\hline
\end{tabular}

\subsection{Registration Phase}

The registration phase involves the following operations:

Step R1. $U_{i}$ chooses his/her identity $I D_{i}$, password $P W_{i}$, and a random number $b$.

Step R2. $U_{i} \Rightarrow S:\left\{I D_{i}, h\left(P W_{i}\right), h\left(b \oplus P W_{i} \oplus I D_{i}\right)\right\}$.

Step $R 3$. On receiving the registration message from $U_{i}$, the server $S$ creates a new entry $\left(h\left(I D_{i}\right), I D_{i}, m\right)$ with the value $m=0$ for $U_{i}$ in the backend database, or sets $m=m+1$ in the existing entry. Then, $S$ computes $E I D=I D \| m, P=h(E I D$ $\oplus x), R=P \oplus h\left(b \oplus P W_{i} \oplus I D_{i}\right)$ and $V=h\left(P \oplus h\left(P W_{i}\right)\right)$.

Step R4. $S \Rightarrow U_{i}$ : A smart card containing security parameters $\{V, R, h(\cdot)\}$.

Step R5. $U_{i}$ enters $b$ into his/her smart card.

\subsection{Login Phase}

When $U_{i}$ wants to login to $S$, the following operations will be performed:

Step L1. $U_{i}$ inserts his/her smart card into the card reader, and inputs $I D_{i}$ and $P W_{i}$.

Step L2. The smart card computes $C_{1}=R \oplus h\left(b \oplus P W_{i} \oplus I D_{i}\right)$ and $C_{2}=h\left(C_{1} \oplus T_{u}\right)$, where $T_{u}$ is the current timestamp on user side.

Step L3. $U_{i} \rightarrow S:\left\{C_{2}, h\left(I D_{i}\right), T_{u}\right\}$.

\subsection{Verification Phase}

After receiving the login request from user $U_{i}, S$ performs the following operations:

Step V1. S first checks the validity of $h\left(I D_{i}\right)$ and $T_{u}$ computes $E I D=I D_{i} \| m$ and $C_{2}{ }^{\prime}$ $=h\left(h(E I D \oplus x) \oplus T_{u}\right)$, and then compares the computed $C_{2}^{\prime}$ with the received $C_{2}$. If they are equal, $S$ computes $C_{3}=h\left(h(E I D \oplus x) \oplus \mathrm{h}\left(T_{s}\right)\right)$ and session key $S K=h\left(h(E I D \oplus x) \oplus I D_{i} \oplus I D_{S} \oplus T_{\mathrm{s}}\right)$, where $I D_{S}$ denotes the identity of $S$. Otherwise, $S$ rejects the request.

Step V2. $S \rightarrow U_{i}:\left\{C_{3}, T_{s}\right\}$.

Step V3. Upon receiving the reply message, $U_{i}$ checks the validity of $T_{u}$. If the verification fails, $U_{i}$ terminates the session. Then, $U_{i}$ computes $C_{3}^{\prime}=h\left(C_{1} \oplus\right.$ 
$h\left(T_{s}\right)$ ), and then compares the computed $C_{3}^{\prime}$ with the received $C_{3}$. If the verification holds, $U_{i}$ computes $S K=h\left(h(E I D \oplus x) \oplus I D_{i} \oplus I D_{S} \oplus T_{\mathrm{s}}\right)$. Otherwise, $U_{i}$ terminates the session.

Step V4. After authenticating each other, $U_{i}$ and $S$ use the same session key $S K$ to secure ensuing data communications.

\subsection{Password Change Phase}

When $U_{i}$ wants to change the old password $P W_{i}$ to the new password $P W_{i}^{\text {new }}$, the following operations will be involved:

Step P1. $U_{i}$ insert his/her own smart card into card reader, keys $I D_{i}$ and $P W_{i}$.

Step $P 2$. The smart card computes $P=R \oplus h\left(b \oplus P W_{i} \oplus I D_{i}\right)$ and $V^{*}=h\left(P \oplus h\left(P W_{i}\right)\right)$, and checks whether $V^{*}$ equals $V$. If the verification fails, smart card rejects.

Step P3. $U_{i}$ keys his/her new password $P W_{i}^{\text {new }}$.

Step $P 4$. The smart card computes $R^{\text {new }}=P \oplus h\left(b \oplus P W_{i}^{\text {new }} \oplus I D_{i}\right)$ and $V^{\text {new }}=h(P \oplus$ $\left.h\left(P W_{i}^{\text {new }}\right)\right)$, and updates $R$ and $V$ with the new $R^{\text {new }}$ and $V^{\text {new }}$ respectively.

\section{Cryptanalysis of Yeh et al.'s Scheme}

There are two assumptions explicitly made in Yeh et al.'s scheme [18]:

(i) The adversary $\mathcal{A}$ has total control over the communication channel between the user $U$ and the remote server $S$. In other words, the adversary can insert, delete, alter, or intercept any messages transmitted in the channel.

(ii) The secret parameters stored in the smart card could be extracted out once a legitimate user's card is somehow (e.g. stolen or picked up) obtained by $\mathcal{A}$.

Note that the above two assumptions, which are also made in the latest works $[7,9,10]$, are indeed reasonable: (1) Assumption $i$ is accordant with the common adversary model introduced in Section 1; and (2) Assumption ii is also practical in consideration of the state-of-art side-channel attack techniques [12-14]. In the following discussions of the security flaws of Yeh et al.'s scheme, based on the above two assumptions, we assume that $\mathcal{A}$ can extract the secret values $\{V, R, b\}$ stored in the legitimate user's smart card, and the attacker can also intercept or block the login request message $\left\{C_{2}, h\left(I D_{i}\right), T_{u}\right\}$ from $U_{i}$ and the reply message $\left\{C_{3}, T_{\mathrm{s}}\right\}$ from $S$.

As described in Yeh et al.'s scheme, mainly two countermeasures are employed to remedy the identified flaws in Hsiang and Shih's scheme: (1) user's ID is concealed by use of a non-invertible hash function to double the difficulty of mounting an offline password guessing attack; (2) a session key is agreed to resist against server impersonation attack. However, as will be shown in the following, the first countermeasure is not effective enough, and the later one lacks key security considerations yet.

\subsection{Offline Password Guessing Attack}

A remote user authentication scheme vulnerable to the offline password guessing attack must satisfy the two conditions: the user's password is weak, and there exists a 
piece of password-related information used as a comparison target for password guessing. In Yeh et al.'s scheme, a user is allowed to choose his/her own password $P W$ at will during the registration and password change phases; the user usually tends to select a password, e.g., his phone number or birthday, which is easily remembered for his convenience. Hence, these easy-to-remember passwords, called weak passwords [19], have low entropy and thus are potentially vulnerable to offline password guessing attack. Inevitably, user's $I D$, chose by the user in the same way with $P W$ as described in the scheme, is exposed to the same threat.

Let us consider the following scenarios. In case a legitimate user $U_{i}$ 's smart card is stolen by an adversary $\mathcal{A}$, and the stored secret values such as $R, V$ and $b$ can be extracted. With a previously eavesdropped message $\left\{C_{2}, h\left(I D_{i}\right), T_{u}\right\}, \mathcal{A}$ can acquire $U_{i}$ 's password $P W_{i}$ by performing the following malicious attack procedure:

Step 1. Guesses all possible values $I D_{i}{ }^{*}$ of $U_{i}$ 's identity, and compares the value of $h\left(I D_{i}^{*}\right)$ with $h\left(I D_{i}\right)$. If the computed $h\left(I D_{i}^{*}\right)$ equals the intercepted $h\left(I D_{i}\right)$, it implies $I D_{i}{ }^{*}=I D_{i}$ and $U_{i}$ 's identity is found, and proceeds to Step 2.

Step 2. Guesses the value of $P W_{i}$ to be $P W_{i}{ }^{*}$ from the password space $\mathcal{D}$.

Step 3. Computes $C_{1}{ }^{*}=R \oplus h\left(b \oplus P W_{i}^{*} \oplus I D_{i}\right)$, where the value of $b$ and $R$ are revealed from the smart card and the value of $I D_{i}$ is obtained through Step 1.

Step 4. Computes $C_{2}{ }^{*}=h\left(C_{1}{ }^{*} \oplus T_{u}\right)$, as $T_{u}$ is previously intercepted.

Step 5. Verifies the correctness of $P W_{i}^{*}$ by checking if $C_{2}{ }^{*}$ equals the intercepted $C_{2}$.

Step 6. Repeats Steps 2, 3, 4 and 5 of this phase until the correct value of $P W_{i}$ is found.

Since the size of password dictionary, i.e. $|\mathcal{D}|$, often is very limited, the above attack procedure can be completed in polynomial time. Halevi and Krawczyk [20] have proved that, under the Dolev-Yao adversary model [11], no password protocol can be free from offline password guessing attack if the public-key techniques are not employed. Therefore, the feasible solution is to reduce the success probability of this attack. Following this principle, Yeh et al.'s scheme thwarts this threat to nearly half success probability as compared to that of Hsiang and Shih's original scheme, which can be easily confirmed from the above attack procedure.

However, we have found that, some minor technical modifications to Yeh et al.'s scheme can quadratically but not linearly reduce the success possibility of this attack. Due to space constraints, we do not give the complete remedy here, and recommend readers to refer the literature [10] for details. The idea of the remedy is not particularly complicated: whenever $P W_{i}$ appears, it is concatenated with the identity $I D_{i}$, while $I D_{i}$ is concealed in dynamic-ID(s). Therefore, the mechanism employed by Yeh et al. to resist against offline password guessing attack is not effective enough as minor revision may thwart this threat to a more desirable extent.

\subsection{Key-Compromise Impersonation Attack}

In the case of key-compromise impersonation, the question is whether the knowledge of a communicating party $A$ 's private key allows a malicious attacker $\mathcal{A}$ not only to impersonate $A$ to others but also to impersonate other uncorrupted parties to $A$. Schemes that prevent this kind of reverse impersonation are said to withstand key-compromise impersonation attack. 
Suppose the long-term secret key $x$ of the server $S$ is leaked out by accident or intentionally stolen by the adversary $\mathcal{A}$. Once the value of $x$ is obtained, with previously intercepted $h\left(I D_{i}\right)$ transmitted in $U_{i}^{\prime}$ s authentication process, $\mathcal{A}$ can impersonate the legitimate user $U_{i}$ through the following method:

Step 1. Guesses $U_{i}$ 's identity to be $I D_{i}{ }^{*}$ from a dictionary of all possible 'weak' identities, and verify the guess by checking whether $h\left(I D_{i}^{*}\right)$ equals $h\left(I D_{i}\right)$.

Step 2. Assumes $m=0$, where $m$ denotes the re-registration times of $U_{i}$.

Step 3. Computes $E I D=I D_{i} \| m$ and $P=h(E I D \oplus x)$, where $I D_{i}$ is derived through Step 1 and $x$ has also been learned.

Step 4. Let $C_{1}=P$ and $C_{2}=h\left(C_{1} \oplus T_{m}\right)$, where $T_{m}$ is the current timestamp.

Step 5. Sends the fabricated login request $\left\{C_{2}, h\left(I D_{i}\right), T_{m}\right\}$ to server $S$.

Step 6. Waits for the reply for a reasonable interval. If no response comes, set $m=m+1$ and goes back to Step 3, else proceeds to the next step.

Step 7. Receives the reply $\left\{C_{3}, T_{s}\right\}$ from server $S$ and computes the session key $S K=$ $h\left(h(E I D \oplus x) \oplus I D_{i} \oplus I D_{S} \oplus T_{\mathrm{s}}\right)$.

Since the value of $m$, i.e. the re-registration times of $U_{i}$, should be very limited in common practice, at most a few dozen, the iteration of the above procedure will come to an end very quickly. The rest of the question is whether Step 1 can be completed in polynomial time. In Yeh et al.'s scheme, a user is allowed to choose his/her own identity $I D$ at will during the registration and password change phases; the user usually tends to select an identity that is human-memorable short strings but not highentropy keys. In other words, they are chosen from the dictionaries of small size. Therefore, the above attack is feasible.

\subsection{Failure to Achieve Forward Secrecy}

As with resistance to key-compromise impersonation attack, the property of forward secrecy is also concerned with limiting the effects of eventual failures, in case the disclosure of server's long-term private keys. Let us consider the following scenarios. Suppose the server $S$ 's long-term private key $x$ is leaked out by accident or intentionally stolen by an adversary $\mathcal{A}$. Once $x$ is obtained, with previously intercepted messages $\left\{h\left(I D_{i}\right), T_{s}^{j}, C_{3}{ }^{j}\right\}$ transmitted during any one of $U_{i}$ 's authenti-cation process (without loss of generality, assume it is $U_{i}^{\prime}$ 's $j$ th authentication process), $\mathcal{A}$ can derive the session key $S K^{j}$ of $S$ and $U_{i}$ 's jth encrypted communication through the following method:

Step 1. Guesses $U_{i}$ 's identity to be $I D_{i}{ }^{*}$ from a dictionary of all possible 'weak' identities, and verify the guess by checking whether $h\left(I D_{i}^{*}\right)$ equals $h\left(I D_{i}\right)$.

Step 2. Assumes $m=0$, where $m$ denotes the re-registration times of $U_{i}$.

Step 3. Computes $E I D=I D_{i} \| m$ and $C_{3}^{j *}=h\left(h(E I D \oplus x) \oplus h\left(T_{s}^{j}\right)\right)$, where $I D_{i}$ is derived through Step 1 and $x$ has also been learned.

Step 4. Compares $C_{3}^{j *}$ with the intercepted $C_{3}^{j}$, this equivalence implies the correct value of $m$ is found. Otherwise, sets $m=m+1$ and goes back to Step 3 .

Step 5. Computes $S K^{j}=h\left(h(E I D \oplus x) \oplus I D_{i} \oplus I D_{S} \oplus T_{s}\right)$. 
The computation complexity of Step 1, Step 3 and Step 4 has been analyzed in Section 3.2, and it's evident that the whole procedure described above can be completed in polynomial time. Once the session key $S K^{j}$ is obtained, the entire $j$ th session will become completely insecure. Consequently, the property of forward secrecy is not provided in Yeh et al.'s scheme, while the provision of forward secrecy is a basic requirement for a secure key agreement scheme.

\subsection{No Provision of User Anonymity}

In Yeh et al.'s scheme, the user $U_{i}^{\prime}$ 's identity $I D_{i}$ is wrapped up in hashing, i.e. $h\left(I D_{i}\right)$, which is static and specific to user $U_{i}$ in all the transaction sessions, an adversary can easily obtain the hashed identity of this communicating client once the login messages were eavesdropped, and hence, different login request messages belonging to the same user can be traced out and may be interlinked to derive some secret information related to the user. Furthermore, $U_{i}$ 's identity $I D_{i}$ may be derived from $h\left(I D_{i}\right)$ through the method introduced in Section 3.1. Hence, user anonymity is not preserved.

\subsection{Some Practical Pitfalls}

In the registration phase, $U_{i}$ 's password $P W_{i}$ is just submitted in a hashed form to $S$, and thus it can be easily derived by $S$. If $U_{i}$ uses this $P W_{i}$ to access several servers for his/her convenience, the insider of $S$ can impersonate $U_{i}$ to access other servers. Hence, it's an insecure factor to commit just a hashed password to the server.

Another pitfall in Yeh's scheme is the slow wrong password detection [15]. If $U_{i}$ inputs a wrong password by mistake, this wrong password will be only detected by the remote system in the verification phase. Therefore, their scheme is slow to detect the user's wrongly input password.

\section{Conclusion}

Smart card-based password authentication technology has been widely deployed in various kinds of security-critical applications, and careful security considerations should be taken into account when designing such schemes. In this paper, we have shown that Yeh et al.'s scheme still suffers from the offline password guessing attack and key-compromise impersonation attack. In addition, their scheme fails to provide the property of forward secrecy and user anonymity. Some other minor defects have also been found. In conclusion, although Yeh et al.'s scheme has many attractive features, it, in fact, does not provide all of the security properties that they claimed and only radical revisions of the protocol can possibly eliminate the identified defects. Therefore, the scheme under study is not recommended for practical application.

Acknowledgements. This research was supported by the National Natural Science Foundation of China (NSFC) under Grants No. 61170241 and No. 61073042. 


\section{References}

1. Vicente, A.G., Munoz, I.B., Galilea, J.L.L., del Toro, P.A.R.: Remote automation laboratory using a cluster of virtual machines. IEEE Transactions on Industrial Electronics 57(10), 3276-3283 (2010)

2. Barolli, L., Xhafa, F.: JXTA-OVERLAY: A P2P platform for distributed, collaborative and ubiquitous computing. IEEE Transactions on Industrial Electronics 58(6), 2163-2172 (2010)

3. Lamport, L.: Password authentication with insecure communication. Communications of the ACM 24(11), 770-772 (1981)

4. Hailer, N.M.: The S/Key One-time Password System. In: Proceedings of the Symposium on Network and Distributed System Security, pp. 151-158. IEEE Press, New York (1994)

5. Chang, C.C., Wu, T.C.: Remote password authentication with smart cards. IEE Proceedings-E 138(3), 165-168 (1993)

6. Yoon, E.J., Ryu, E.K., Yoo, K.Y.: Further improvement of an efficient password based remote user authentication scheme using smart cards. IEEE Transactions on Consumer Electronics 50(2), 612-614 (2004)

7. Yang, G., Wong, D.S., Wang, H., Deng, X.: Two-factor mutual authentication based on smart cards and password. Journal of Computer and System Sciences 74(7), 1160-1172 (2008)

8. Hsiang, H.C., Shih, W.K.: Weaknesses and improvements of the Yoon-Ryu-Yoo remote user authentication scheme using smart cards. Computer Communications 32(4), 649-652 (2009)

9. Sood, S.K., Sarje, A.K., Singh, K.: An improvement of Hsiang-Shih's authentication scheme using smart cards. In: Proceedings of ICWET 2010, pp. 19-25. ACM Press, New York (2010)

10. Ma, C.-G., Wang, D., Zhang, Q.-M.: Cryptanalysis and Improvement of Sood et al.'s Dynamic ID-Based Authentication Scheme. In: Ramanujam, R., Ramaswamy, S. (eds.) ICDCIT 2012. LNCS, vol. 7154, pp. 141-152. Springer, Heidelberg (2012)

11. Dolev, D., Yao, A.C.: On the security of public key protocols. IEEE Transactions on Information Theory 29(2), 198-208 (1983)

12. Kocher, P., Jaffe, J., Jun, B.: Differential Power Analysis. In: Wiener, M. (ed.) CRYPTO 1999. LNCS, vol. 1666, pp. 388-397. Springer, Heidelberg (1999)

13. Messerges, T.S., Dabbish, E.A., Sloan, R.H.: Examining Smart-Card Security under the Threat of Power Analysis Attacks. IEEE Transactions on Computers 51(5), 541-552 (2002)

14. Mangard, S., Oswald, E., Standaert, F.X.: One for all-all for one: unifying standard differential power analysis attacks. IET Information Security 5(2), 100-110 (2011)

15. Tsai, C., Lee, C., Hwang, M.: Password authentication schemes: current status and key issues. International Journal of Network Security 3(2), 101-115 (2006)

16. Blake-Wilson, S., Johnson, D., Menezes, A.: Key Agreement Protocols and Their Security Analysis. In: Darnell, M.J. (ed.) Cryptography and Coding 1997. LNCS, vol. 1355, pp. 3045. Springer, Heidelberg (1997)

17. Krawczyk, H.: HMQV: A High-Performance Secure Diffie-Hellman Protocol. In: Shoup, V. (ed.) CRYPTO 2005. LNCS, vol. 3621, pp. 546-566. Springer, Heidelberg (2005)

18. Yeh, K.H., Su, C.H., Lo, N.W.: Two robust remote user authentication protocols using smart cards. Journal of Systems and Software 83(12), 2556-2565 (2010)

19. Klein, D.V.: Foiling the Cracker: A Survey of, and Improvements to, Password Security. In: 2nd USENIX Security Workshop, pp. 5-14. USENIX Association, Portland (1990)

20. Halevi, S., Krawczyk, H.: Public-key cryptography and password protocols. ACM Transactions on Information and System Security 2(3), 230-268 (1999) 